\title{
Bacterial Gall of Loropetalum chinense caused by Pseudomonas amygdali pv. loropetali pv. nov.
}

Carrie Lapaire Harmon, ${ }^{\dagger}$ Sujan Timilsina, and John Bonkowski, University of Florida Department of Plant Pathology, Gainesville, FL; Debra D. Jones and Xiaoan Sun, Florida Department of Agriculture and Consumer Services, Division of Plant Industry, Gainesville, FL; Gary E. Vallad, University of Florida Department of Plant Pathology, GCREC, Wimauma, FL; Laura Ramos Sepulveda and Carolee Bull, The Pennsylvania State University, University Park, PA; and Jeffrey B. Jones, ${ }^{\dagger}$ University of Florida Department of Plant Pathology, Gainesville, FL

\begin{abstract}
In 2012, stem gall samples on Loropetalum chinense were sent to Florida diagnostic labs from Alabama and Florida nurseries. A fluorescent pseudomonad was consistently isolated from the galls. The organism was originally identified in Alabama based on 16S rRNA sequencing as Pseudomonas savastanoi, which causes a production-limiting disease of olive. The loropetalum strains and reference strains were compared using LOPAT, Biolog, fatty acid analysis, multilocus sequence analysis (MLSA), and pathogenicity tests. The LOPAT tests placed the loropetalum strains within Pseudomonas syringae. Biolog and fatty acid analysis placed the strains in various pathovars of $P$. syringae and $P$. savastanoi,

respectively. MLSA of a set of housekeeping genes separated the loropetalum strains from the olive knot-inducing strains. Our work indicates there is a need to use more tests than $16 \mathrm{~S}$ rRNA to accurately diagnose new bacterial diseases. In pathogenicity tests, the loropetalum strains produced galls only on loropetalum, but not on olive, mandevilla, or almond, indicating this strain is not a threat to the olive industry. Based on the pathogenicity assays and molecular tests, loropetalum strains represent a distinct and new pathovar, $P$. amygdali pv. loropetali pv. nov., for which the strain PDC13-208 (= DSMZ $105780^{\mathrm{PT}}$ ) has been designated as the pathotype strain.
\end{abstract}

The Pseudomonas syringae complex includes 10 Pseudomonas species and at least 60 pathovars (Young 2010). There is some debate regarding the classification of Pseudomonas species and the subspecies/pathovars, including the olive strain of the $P$. syringae/ savastanoi bacterium (Gardan et al. 1992; Hwang et al. 2005; Penyalver et al. 2000; Ramos et al. 2012; Sarkar and Guttman 2004). Historically, nomenclature has been confused by the use of synonyms for the same pathogen found in different locations and classified as different species (Bull and Koike 2015; Young 2010). Differentiation of Pseudomonas strains is further complicated by a lack of understanding of fundamental physiology and metabolism (Young 2010). Additionally, sequencing of 16S rRNA, commonly used to differentiate bacteria in diagnostic laboratories, cannot resolve differences between many Pseudomonas species (Janda and Abbott 2007; Young 2010). This inability to easily differentiate taxa despite the genomic diversity has led to confusion concerning the taxonomy of the $P$. syringae complex (Young 2010).

$P$. syringae sensu lato, based on genomic comparisons using DNA-DNA hybridization, consists of nine distinct genomospecies within this pathogen complex (Gardan et al. 1999). Bull et al. (2011) determined that phylogenies based on four housekeeping genes, including rpoD, were useful for delineating eight genomospecies. Gardan et al. (1999) placed $P$. savastanoi in genomospecies 2, indicating that it is a synonym of $P$. amygdali, as are $P$. ficuserectae and $P$. meliae. Additionally, Marques et al. (2008), using rep-PCR for comparisons, demonstrated that $P$. tremae was also a synonym of $P$. amygdali. Likewise, analysis with rpoD confirmed that $P$. savastanoi, $P$. ficuserectae, $P$. meliae, and $P$. tremae are members of genomospecies 2 (also called phylogroup 3 ) and are thus synonyms

${ }^{\dagger}$ Corresponding authors: Carrie Lapaire Harmon, E-mail: clharmon@ufl.edu; and Jeffrey Jones, E-mail: jbjones@ufl.edu

This research was supported in part by the University of Florida IFAS Plant Diagnostic Center and projects funded by the USDA, including the USDANIFA Southern Plant Diagnostic Network and the USDA Agriculture and Food Research Initiative Plant Biosecurity Program.

Accepted for publication 12 October 2017.

C) 2018 The American Phytopathological Society of $P$. amygdali and distinct from $P$. syringae sensu stricto and the other genomospecies (Parkinson et al. 2011).

Loropetalum chinense, commonly known as loropetalum or fringe flower, is a common woody ornamental in the Mid- and Deep South that is propagated by cuttings. In 2012, we received loropetalum samples from Alabama and Florida in diagnostic laboratories in Florida and isolated a fluorescent pseudomonad. In 2013, Conner et al. (2013) reported on a bacterial gall on loropetalum that was identified as $P$. savastanoi based on phenotypic characteristics along with sequence analysis of partial sequences of the 16S rRNA gene. As a result of the concern of $P$. savastanoi potentially entering the fledgling olive industry in Florida, these fluorescent pseudomonads isolated from galls on loropetalum plants were analyzed using a polyphasic approach to characterize the loropetalum strains from both Alabama and Florida. P. savastanoi pv. savastanoi, the causal agent of olive knot disease, is a member of the $P$. syringae complex. P. savastanoi $\mathrm{pv}$. savastanoi exhibits variation in host range and virulence and is both phenotypically and genotypically heterogeneous (Ramos et al. 2012). As a result of the lower sequence identity with the partial sequence of the $P$. savastanoi type strain than with $P$. syringae pv. eriobotryae, it was necessary to more precisely define the taxonomic position of the strains. The loropetalum and reference strains were compared using LOPAT, Biolog, fatty acid analysis, multilocus sequence analysis (MLSA), and pathogenicity tests. Results from the LOPAT tests placed the loropetalum strains in P. syringae, while Biolog and fatty acid analysis tests placed the strains in various pathovars of $P$. syringae and $P$. savastanoi, respectively. Based on partial sequence analysis of the $16 \mathrm{~S}$ rRNA, a representative loropetalum strain had $100 \%$ sequence identity with $P$. syringae pv. eriobotryae and $99.9 \%$ sequence identity with $P$. meliae (a synonym of $P$. amygdali), $P$. savastanoi pv. savastanoi, and $P$. amygdali. MLSAs of a set of housekeeping genes placed the loropetalum strains in genomospecies 2-Group A, which also contains all the species and pathovar eriobotryae mentioned above. In pathogenicity tests in which all the pathotypes in genomospecies 2-Group A and four pathotypes in Group B were compared, the loropetalum strains produced galls only on loropetalum, but not on olive, mandevilla, or almond. Sequence analysis of $i a a L$ placed the loropetalum strains separate from $P$. savastanoi pv. savastano $i$ and other gall-forming pseudomonads including the $P$. amygdali type strain on almond, LMG $13184^{\mathrm{T}}$.

We determined that the loropetalum strains are members of genomospecies 2 and cluster more closely with the type strain of 
P. amygdali (LMG $\left.13184^{\mathrm{T}}\right)$ than with P. savastanoi pv. savastanoi $\left(\right.$ LMG $2209^{\mathrm{PT}}$ ) or $P$. syringae pv. eriobotryae (LMG $2184^{\mathrm{PT}}$ ). Here we propose a new pathovar, $P$. amygdali pv. loropetali pv. nov. $\left(\mathrm{DSMZ} \mathrm{XXXX} \mathrm{XT}^{\mathrm{PT}}\right)$, as the agent causing galls on loropetalum.

\section{Materials and Methods}

Bacterial strains. Bacterial strains analyzed included the following: 1) type and pathotype strains (Table 1) obtained from BCCM/LMG (Belgian Coordinated Collection of Microorganisms/Laboratory for Microbiology, Ghent University, Belgium), NCPPB (National Collection of Plant Pathogenic Bacteria), GRBio (The Global Registry of Biodiversity Repositories), and the ICMP (International Collection of Microorganisms from Plants)/Landcare Research - Manaaki Whenua; 2) 32 strains isolated from loropetalum obtained from the Florida Department of Agriculture and Consumer Services, Division of Plant Industry (FDACS, DPI); 3) one strain isolated from a routine diagnostic sample submitted to the UF/IFAS Plant Diagnostic Center from a nursery in Alabama; and 4) one strain provided by K. Conner
(Plant Disease Clinic, Auburn University, Auburn, AL) to represent the strains studied in 2012 (Conner et al. 2013). All strains were stored at $-80^{\circ} \mathrm{C}$, and were grown on nutrient agar (BBL, Becton Dickinson and Co., Cockeysville, MD) at $27^{\circ} \mathrm{C}$, except $P$. meliae $\left(\right.$ LMG $\left.02220^{\mathrm{T}}\right)$, $P$. tremae $\left(\mathrm{LMG} 22121^{\mathrm{T}}\right)$, and $P$. amygdali $(\mathrm{LMG}$ $13184^{\mathrm{T}}$ ), which were grown at $27^{\circ} \mathrm{C}$ on medium 51 as described and recommended by BCCM (http://bccm.belspo.be/).

Biochemical and physiological tests. All strains were tested for fluorescence on King's medium B (KMB) (Schaad et al. 2001). Each strain was streaked onto a $\mathrm{KMB}$ agar plate and incubated at $27^{\circ} \mathrm{C}$ for up to $72 \mathrm{~h}$. Fluorescence was tested by exposing the plate to long wavelength UV light. All strains were analyzed with Biolog 96-well plates using a plate reader (Emax Endpoint microplate reader, Molecular Devices LLC, Sunnyvale, CA) and the Biolog Microbial ID GenIII Microlog plates, software package, and library (Biolog, Haward, CA). Fatty acid analysis of these strains was performed using the MIDI Sherlock Microbial Identification System. Dendrograms were developed with the strains including several library

Table 1. Bacterial strains used in this study

\begin{tabular}{|c|c|c|c|c|}
\hline Strain designation ${ }^{\mathbf{a}}$ & Bacterial species & Host & Source & Biolog results \\
\hline LMG 2184 & $\begin{array}{l}\text { Pseudomonas syringae pv. } \\
\text { eriobotryae }\end{array}$ & Eriobotrya japonica & BCCM/LMG & P. syringae pv. syringae \\
\hline LMG $2209^{\mathrm{PT}}$ & P. savastanoi pv. savastanoi & Olea europaea & BCCM/LMG & P. savastanoi pv. nerii \\
\hline LMG $2245^{\mathrm{PT}}$ & P. savastanoi pv. phaseolicola & Phaseolus vulgaris & BCCM/LMG & P. syringae pv. phaseolicola \\
\hline LMG $2220^{\mathrm{T}}$ & P. meliae & Melia azedarach & $\mathrm{BCCM} / \mathrm{LMG}$ & P. syringae pv. phaseolicola \\
\hline LMG 5385 & P. savastanoi pv. nerii & Nerium oleander & BCCM/LMG & P. savastanoi pv. nerii \\
\hline LMG $13184^{\mathrm{T}}\left(=\mathrm{CFBP} 3205^{\mathrm{T}}\right)$ & P. amygdali & Prunus dulcis & BCCM/LMG & P. syringae pv. delphinii \\
\hline LMG $22121^{\mathrm{T}}$ & P. tremae & Trema orientalis & $\mathrm{BCCM} / \mathrm{LMG}$ & P. syringae pv. glycinea \\
\hline LMG5066 $^{\text {PT }}$ & P. savastanoi pv. glycinea & Glycine $\max$ & BCCM/LMG & $\mathrm{ND}^{\mathrm{b}}$ \\
\hline LMG5694 & P. ficuserectae & Ficus erecta & BCCM/LMG & ND \\
\hline LMG5541 ${ }^{\text {PT }}$ & P. syringae pv. ciccaronei & Ceratonia siliqua & $\mathrm{BCCM} / \mathrm{LMG}$ & ND \\
\hline LMG5668 ${ }^{\text {PT }}$ & P. syringae pv. myricae & Myrica rubra & BCCM/LMG & ND \\
\hline LMG2349 ${ }^{\mathrm{PT}}$ & P. syringae pv. ulmi & Ulmus sp. & $\mathrm{BCCM} / \mathrm{LMG}$ & ND \\
\hline NCPPB3681 1 PT & P. syringae pv. aesculi & Aesculus indica & NCPPB & ND \\
\hline $\mathrm{NCPPB} 3682^{\mathrm{PT}}$ & P. syringae pv. hibisci & $\begin{array}{l}\text { Hibiscus rosa } \\
\text { sinensis }\end{array}$ & NCPPB & ND \\
\hline NCPPB3618 & P. syringae pv. rhaphiolepidis & $\begin{array}{l}\text { Rhaphiolepis } \\
\text { umbellata }\end{array}$ & NCPPB & ND \\
\hline ICMP11894PT & P. syringae pv. cunninghamiae & $\begin{array}{l}\text { Cunninghamia } \\
\text { lanceolata }\end{array}$ & ICMP & ND \\
\hline MD340a & P. syringae pv. tabaci & Nicotiana tabacum & & ND \\
\hline $\begin{array}{l}\text { PDC13-198, 199, 200, 201, 203, } \\
\begin{array}{l}204,205,206,207,208,209,210 \\
211,212,213,214,215,216,217 \\
218,219,220,221,222,223,224 \\
225,227,228\end{array}\end{array}$ & & $\begin{array}{l}\text { Loropetalum } \\
\text { chinense }\end{array}$ & $\begin{array}{l}\text { FDACS, DPI; Florida } \\
\text { nurseries }\end{array}$ & $\begin{array}{l}\text { P. savastanoi pv. nerii }(15) ; \\
\text { P. syringae pv. phaseolicola }(2) ; \\
\text { pv. fraxini }(7) ; \text { P. savastanoi; } \\
\text { P. agaraci }(3) ; \text { P. syringae } \\
\text { pv. antirrhini }(1) ; \text { P. syringae } \\
\text { pv. delphinii }(1)\end{array}$ \\
\hline PDC12254 & & $\begin{array}{l}\text { Loropetalum } \\
\text { chinense }\end{array}$ & $\begin{array}{l}\text { UF/IFAS PDC; Alabama } \\
\text { nursery }\end{array}$ & P. savastanoi pv. nerii \\
\hline PDL384 & & $\begin{array}{l}\text { Loropetalum } \\
\text { chinense }\end{array}$ & $\begin{array}{l}\text { Auburn PDL; Alabama } \\
\text { nursery }\end{array}$ & ND \\
\hline
\end{tabular}

${ }^{\text {a T }}=$ Type strain, ${ }^{\mathrm{PT}}=$ pathotype strain.

${ }^{\mathrm{b}}$ Not determined.

Table 2. GenBank accession numbers for multilocus sequence alignment study

\begin{tabular}{|c|c|c|c|c|c|c|}
\hline Strains & $16 s^{a}$ & gyrB & gap1 & gltA & rpoD & $i a a L$ \\
\hline PDC13-206 & NA & KM593940 & KM593956 & KM593966 & KM593986 & KM593976 \\
\hline $\mathrm{PDC} 13-208^{\mathrm{PT}}$ & KM593950 & KM593941 & KM593957 & KM593967 & KM593987 & KM593977 \\
\hline PDC13-219 & NS & KM593942 & KM593958 & KM593968 & KM593988 & KM593978 \\
\hline PDC12-254 & NA & KM593943 & KM593959 & KM593969 & KM593989 & KM593979 \\
\hline PDL474 & KM593952 & KM593944 & KM593960 & KM593970 & KM593990 & KM593980 \\
\hline LMG2184 & KM593951 & KM593945 & KM593961 & KM593971 & KM593991 & KM593981 \\
\hline LMG2209 & KM593953 & KM593946 & KM593962 & KM593972 & KM593992 & KM593982 \\
\hline LMG2245 & NA & KM593947 & KM593963 & KM593973 & KM593993 & KM593983 \\
\hline LMG2220 & KM593954 & KM593948 & KM593964 & KM593974 & KM593994 & KM593984 \\
\hline LMG5385 & KM593955 & KM593949 & KM593965 & KM593975 & KM593995 & KM593985 \\
\hline
\end{tabular}

${ }^{\mathrm{a}} \mathrm{NA}=$ Strain was not sequenced for 16s gene, NS = sequence was not submitted to NCBI. 
references (Sherlock version 6.1, TSBA6 database version 6.10, MIDI Labs, Inc., Newark, DE). All strains were subjected to the LOPAT tests, which included assaying for levan production, oxidase reaction, potato rot, arginine dihydrolase activity, and hypersensitive response on tobacco (Schaad et al. 2001). To determine hypersensitivity response, strains were inoculated by infiltrating bacterial suspensions adjusted to $1 \times 10^{8} \mathrm{CFU}$ suspension into 'Hicks' tobacco leaves and 'Bonnie Best' tomato leaves. Inoculations were checked daily for confluent necrosis for up to 3 days.

Pathogenicity tests. Stem inoculations of the Florida strains (PDC13-198 to PDC13-228) onto host plants were conducted by two methods, five to 10 times on a stem of each host type: 1) syringe injection of bacterial suspensions adjusted to $1 \times 10^{8} \mathrm{CFU} / \mathrm{ml}$; or 2 ) insertion of a sterile toothpick dipped in a 24-h culture from a

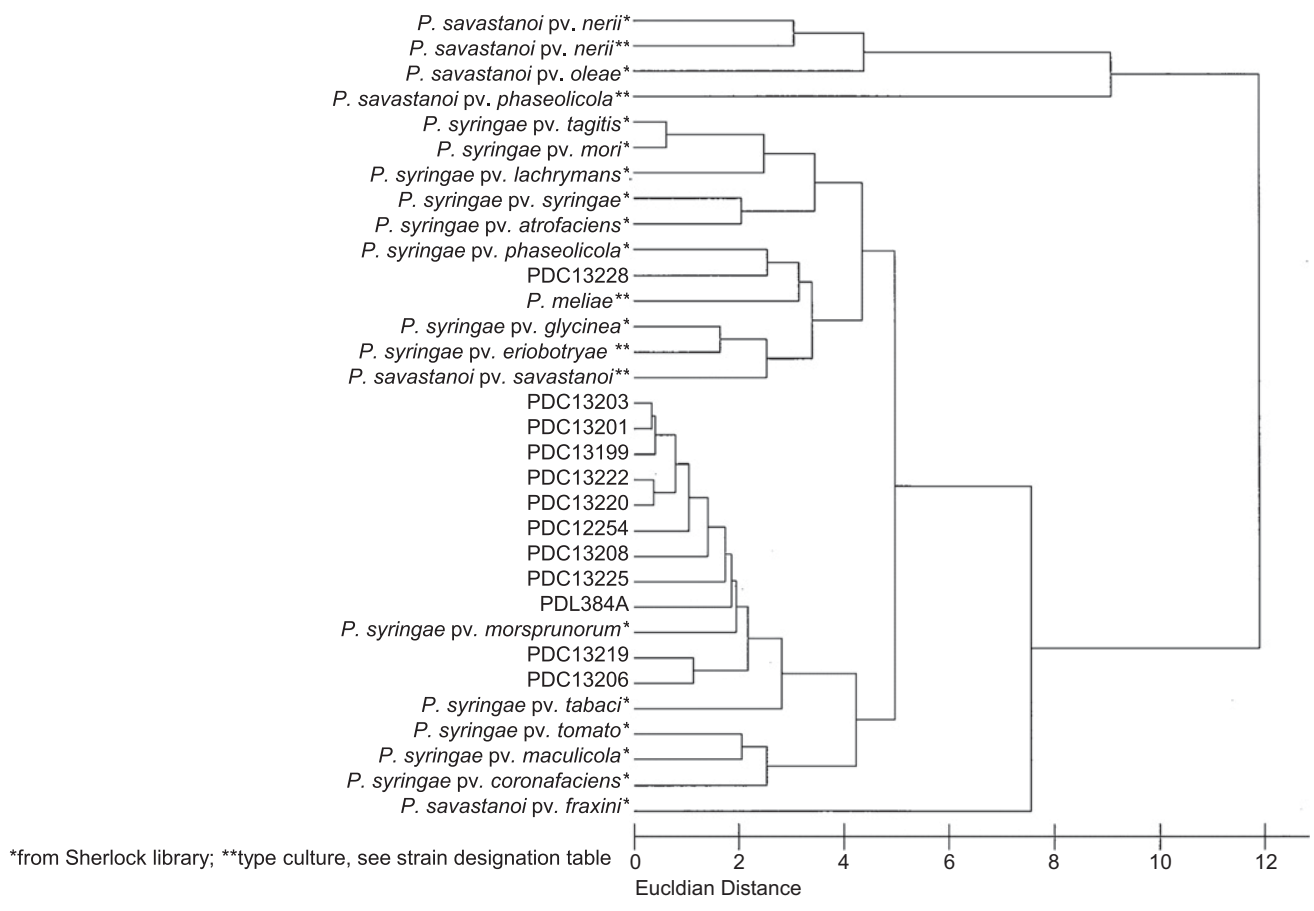

Fig. 1. Dendrogram showing relationships of fluorescent strains isolated from loropetalum and other fluorescent pseudomonads based on fatty acid analysis.

Table 3. Comparison of fatty acid profiles of loropetalum strains with select pseudomonads ${ }^{\mathrm{a}}$

\begin{tabular}{|c|c|c|c|c|c|c|c|}
\hline Fatty acid & LMG $2220^{T}$ & P. amygdali & LMG 2245 ${ }^{\mathrm{PT}}$ & LMG 2184 ${ }^{\mathrm{PT}}$ & LMG 5385 & LMG $2209^{T}$ & Loropetalum strains \\
\hline 10:0 & 0.12 & $\mathrm{TR}^{\mathrm{c}}$ & 0.07 & 0.07 & 0.11 & 0.09 & $0.07(0.03)$ \\
\hline $10: 03 \mathrm{OH}$ & 3.96 & $14.3(4.5)$ & 2.78 & 2.76 & 3.36 & 3.17 & $3.25(0.20)$ \\
\hline $12: 0$ & 5.24 & $5.1(0.4)$ & 4.78 & 4.42 & 4.14 & 4.23 & $4.40(0.25)$ \\
\hline $11: 0$ iso $3 \mathrm{OH}$ & & & 0.48 & & 0.07 & & \\
\hline $13: 0$ iso & & & 0.12 & & & & \\
\hline $13: 1$ at $12-13$ & & & & & 0.07 & & \\
\hline $12: 02 \mathrm{OH}$ & 3.46 & $2.0(0.6)$ & 2.89 & 3.04 & 3.06 & 3.06 & $3.07(0.15)$ \\
\hline $12: 13 \mathrm{OH}$ & & TR & & & & & \\
\hline $12: 03 \mathrm{OH}$ & 5.02 & $4.4(1.0)$ & 4.24 & 4.06 & 4.23 & 3.94 & $4.04(0.13)$ \\
\hline 14:0 & 0.30 & $0.2(0.1)$ & 0.25 & 0.22 & 0.21 & 0.27 & $0.66(0.13)$ \\
\hline Sum In Feature 3 & 33.77 & 14.1 & 29.09 & 35.10 & 28.81 & 33.40 & $37.03(1.29)$ \\
\hline 16:0 & 29.30 & $17.2(4.3)$ & 28.48 & 27.89 & 25.44 & 29.39 & $29.44(0.75)$ \\
\hline Sum In Feature 4 & 0.17 & & & & & & \\
\hline $17: 0$ iso & & & 1.95 & 0.23 & 0.29 & 0.21 & \\
\hline 17:0 cyclo & & $2.6(0.6)$ & 6.51 & 1.11 & 0.50 & 0.60 & $0.45(0.32)$ \\
\hline $17: 00$ & & & & 0.17 & & & \\
\hline $16: 03 \mathrm{OH}$ & 0.58 & $7.9(2.6)$ & & & 0.08 & 0.16 & \\
\hline Sum In Feature 8 & 16.70 & $22.7(2.8)$ & 16.78 & 19.07 & 25.24 & 18.98 & $14.75(1.41)$ \\
\hline $18: 1 \mathrm{w} 5 \mathrm{c}$ & & & & & 0.11 & & $0.09(0.03)$ \\
\hline 18:0 & 0.80 & $0.5(0.1)$ & 0.83 & 1.35 & 3.06 & 1.43 & $1.58(0.29)$ \\
\hline 18:1 w7c 11-methyl & 0.27 & & 0.18 & 0.40 & 1.02 & 0.75 & $0.63(0.15)$ \\
\hline Sum Feature 7 & & $0.4(0.3)$ & 0.34 & 0.11 & & 0.20 & $0.34(0.11)$ \\
\hline 19:0 10-methyl & 0.30 & $4.7(2.4)$ & & & & 0.14 & $0.04(0.02)$ \\
\hline 19:0 cyclo w8c & & $0.2(0.2)$ & 0.22 & & & & \\
\hline \multicolumn{8}{|l|}{ 19:O CYCIO 11-12 } \\
\hline 19:0 cyclo w8c & & $0.2(0.2)$ & & & & & \\
\hline $20: 1 \mathrm{w} 7 \mathrm{c}$ & & & & & 0.21 & & $0.16(0.05)$ \\
\hline
\end{tabular}

a Strains: P. meliae LMG 2220; P. amygdali; P. savastanoi pv. phaseolicola LMG 2245; P. syringae pv. eriobotryae LMG 2184; P. savastanoi pv. savastanoi LMG 5385; P. savastanoi pv. savastanoi LMG 2209; Loropetalum strains (14).

${ }^{\mathrm{b}}$ Fatty acid data for $P$ amygdali were taken from Table 5 in Stead (1992).

c $\mathrm{TR}=$ Trace 
nutrient agar plate into young stems. Host plants included for this study were almond (Prunus dulcis), loropetalum (L. chinense), mandevilla (Mandevilla sanderi), oleander (Nerium oleander), and olive (Olea europaea). Plants were maintained in a greenhouse at the University of Florida. Inoculations of the two Alabama strains (G12-254 and PDL384) and the six LMG strains (LMG $2184^{\mathrm{PT}}, 2209^{\mathrm{PT}}$, $2245^{\mathrm{PT}}, 2220^{\mathrm{T}}, 5385^{\mathrm{PT}}, 13184^{\mathrm{T}}$, and $22121^{\mathrm{T}}$ ) were conducted on almond, loropetalum, oleander, and olive in a containment greenhouse at FDACS-DPI, Gainesville, FL. Each strain was inoculated as above. An additional set of pathogenicity experiments were conducted to determine pathogenicity on loropetalum by types and pathotypes within in $P$. amygdali. In these experiments, five loropetalum strains (DSMZ 105780 ${ }^{\mathrm{PT}}$, PDC13-254, PDC13-672, PDC13-206, and PDC13-219) and 13 types and pathotypes from genomospecies 2 were evaluated for gall formation: $P$. savastanoi pv. glycinea (LMG $\left.5066^{\mathrm{PT}}\right)$, P. amygdali $\left(\mathrm{LMG} 13184^{\mathrm{T}}\right)$, P. ficuserectae $\left(\mathrm{LMG} 5694^{\mathrm{T}}\right)$, P. meliae (LMG $\left.2220^{\mathrm{T}}\right)$, P. syringae pv. ciccaronei $\left(\mathrm{LMG} 5541^{\mathrm{PT}}\right)$, $P$. syringae pv. myricae (LMG5668 ${ }^{\mathrm{PT}}$ ), $P$. syringae pv. eriobotryae (LMG $2184^{\mathrm{PT}}$ ), P. syringae pv. ulmi (LMG $2349^{\mathrm{PT}}$ ), P. syringae pv. aesculi (NCPPB $3681^{\mathrm{PT}}$ ), P. syringae pv. hibisci (NCPPB $3682^{\mathrm{PT}}$ ), P. syringae pv. tabaci (NCPPB $1427^{\mathrm{PT}}$ ), P. syringae pv. cunninghamiae (ICMP $11894^{\mathrm{PT}}$ ), and $P$. syringae pv. rhaphiolepidis $\left(\mathrm{NCPPB} 3618^{\mathrm{PT}}\right.$ ). Multiple inoculations (at least three) were made on a single stem by inserting a sterile toothpick coated with a test strain into young stems and then covering the wounds with Parafilm to keep them moist. The assay was carried out in a growth chamber set to $80 \%$ relative humidity and at $27^{\circ} \mathrm{C}$ during $14 \mathrm{~h}$ of light and $25^{\circ} \mathrm{C}$ when the chamber was dark. The experiment was repeated twice with three replicate stems inoculated per strain in each experiment. The results were identical in both experiments. The bacteria were reisolated from galled tissue and a rep-PCR using BOX1A primers was performed to confirm that the strain was the same as originally inoculated. Plants used in this study were grown using clonal propagation of each host type in order to ensure differences in pathogenic effects were a direct result of the strain. Syringe injection of sterile tap water and sterile toothpicks dipped in sterile water served as negative controls when inoculated onto each of the host plant types. Plants were checked weekly for symptom development.

Phylogenetic analysis. Genomic DNA from loropetalum strains PDL384A, PDC13208, and PDC13219 and LMG strains listed in Table 1 was extracted using the Qiagen DNeasy plant mini kit (cat. 69104, Qiagen, Germantown, MD). For 16S rRNA sequencing, approximately $1.4 \mathrm{~kb}$ of the $16 \mathrm{~S}$ rRNA sequence was amplified from DNA of each strain using the universal primers: $63 \mathrm{f}\left(5^{\prime}-\right.$ CAGGCCTAACACATGCAAGTC-3') and reverse primer $1387 \mathrm{r}$ (5'-GGGCGGWGTGTACAAGGC-3') (Marchesi et al. 1998). Given that multiple 16S gene copies are common in Pseudomonas spp.

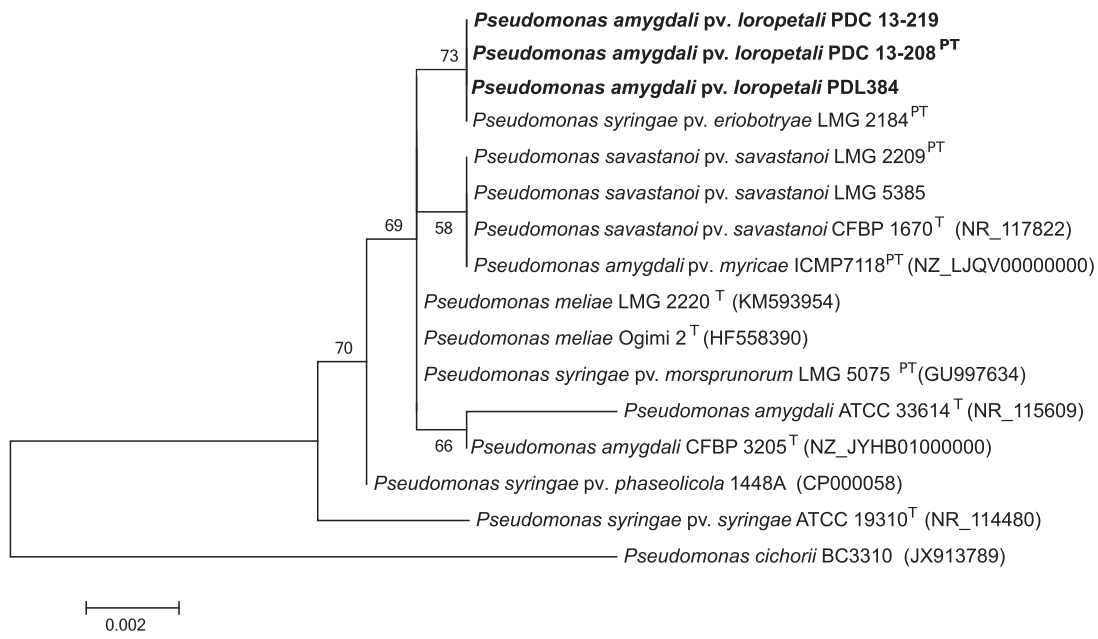

Fig. 2. Phylogenetic distribution of Pseudomonas strains isolated from loropetalum using 16s rRNA sequences. Maximum likelihood phylogenetic tree was constructed based on the sequence available using gamma distributed general time reversible with invariant sites model.

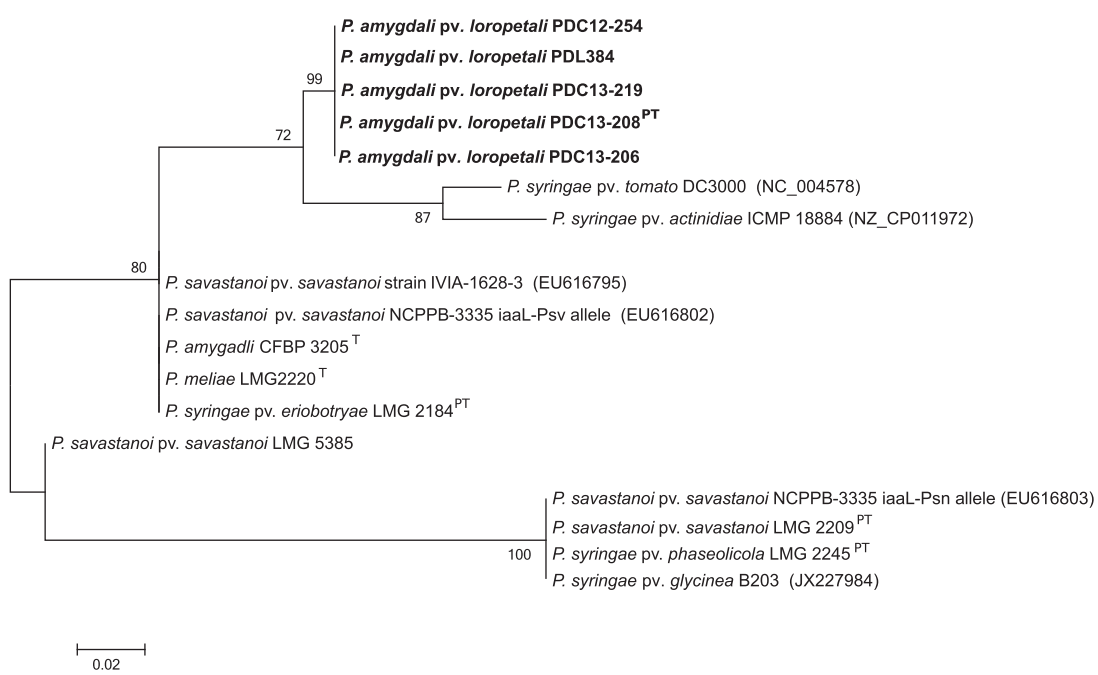

Fig. 3. Maximum likelihood phylogenetic distribution based on iaa genes for Pseudomonas strains isolated from loropetalum. The trees were constructed based on the model described previously. 


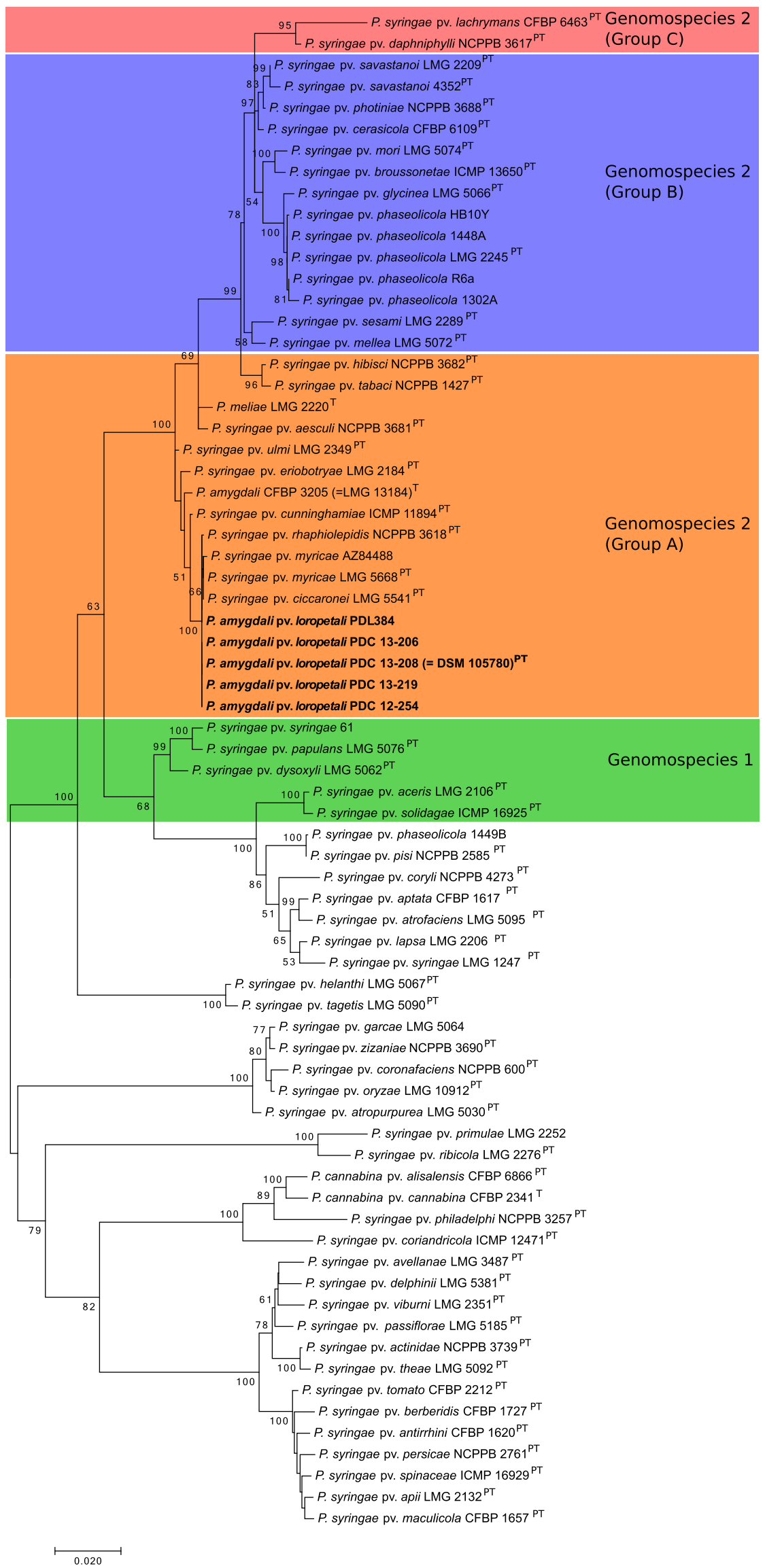

Fig. 4. Phylogenetic distribution of $P$ seudomonas strains isolated from loropetalum using concatenated housekeeping gene sequences. Four conserved housekeeping genes were used, namely gap1, gyrB, gltA, and rpoD. Maximum likelihood phylogenetic distribution tree was constructed based on GTR $+\mathrm{GI}$ model. The housekeeping genes for the loropetalum isolates were found identical to each other and were closely related to group A of genomospecies 2, P. amygdali strains. 
(Coenye and Vandamme 2003), following PCR, the products were cloned into pGEM-T vector and transformed into Escherichia coli before sequencing. The cloned 16S PCR products were sequenced at the University of Florida ICBR core facility.

MLSA for the loropetalum strains and selected strains listed in Table 1 was performed by sequencing four housekeeping genes: gyrB (gyrase B, $507 \mathrm{bp}$ ), rpoD (RNA polymerase sigma $^{70}$ factor, 498 bp), gapl (glyceraldehyde-3-phosphate dehydrogenase A, $476 \mathrm{bp}$ ), and gltA (citrate synthase, $529 \mathrm{bp}$ ), using the MLST schema as described by Hwang et al. (2005). Amplicons were sequenced bidirectionally (Sanger sequencing, Eurofins MWG Operon, Huntsville, AL), aligned, and edited using the MUSCLE tool available within MEGA 6 (Tamura et al. 2013). Edited sequences were combined to obtain a concatenated sequence for each strain. Phylogenetic analysis was performed on concatenated sequences along with $P$. syringae pathovars (Bull et al. 2011) and other selected sequences of $P$. syringae strains from various hosts and locations displaying at most a 0.002 maximum composite pairwise distance between the strains PDL384, PDC13208, and PDC13219 and LMG strains listed in Table 1. Sequences were obtained from the Plant Associated Microbes Database (PAMDB) (http://genome.ppws.vt.edu/cgi-bin/ MLST/home.pl) and whole genome shotgun alignments from GenBank.

The indoleacetic acid gene (iaaL), which is associated with knot and gall development in host plants (Penyalver et al. 2000), is widely distributed within the $P$. syringae complex (Glickmann et al. 1998), and according to Ramos et al. (2012), its phylogeny is generally congruent with the phylogeny determined using housekeeping genes. In order to amplify iaaL, boiled DNA from selected loropetalum strains and LMG strains listed in Table 1 was subjected to PCR using primers IAALF (GGCACCAGCGGCAACATCAA) and IAALR (CGCCCTCGGAACTGCCATAC) (Penyalver et al. 2000). Following amplification, the PCR products of approximately $294 \mathrm{bp}$ were

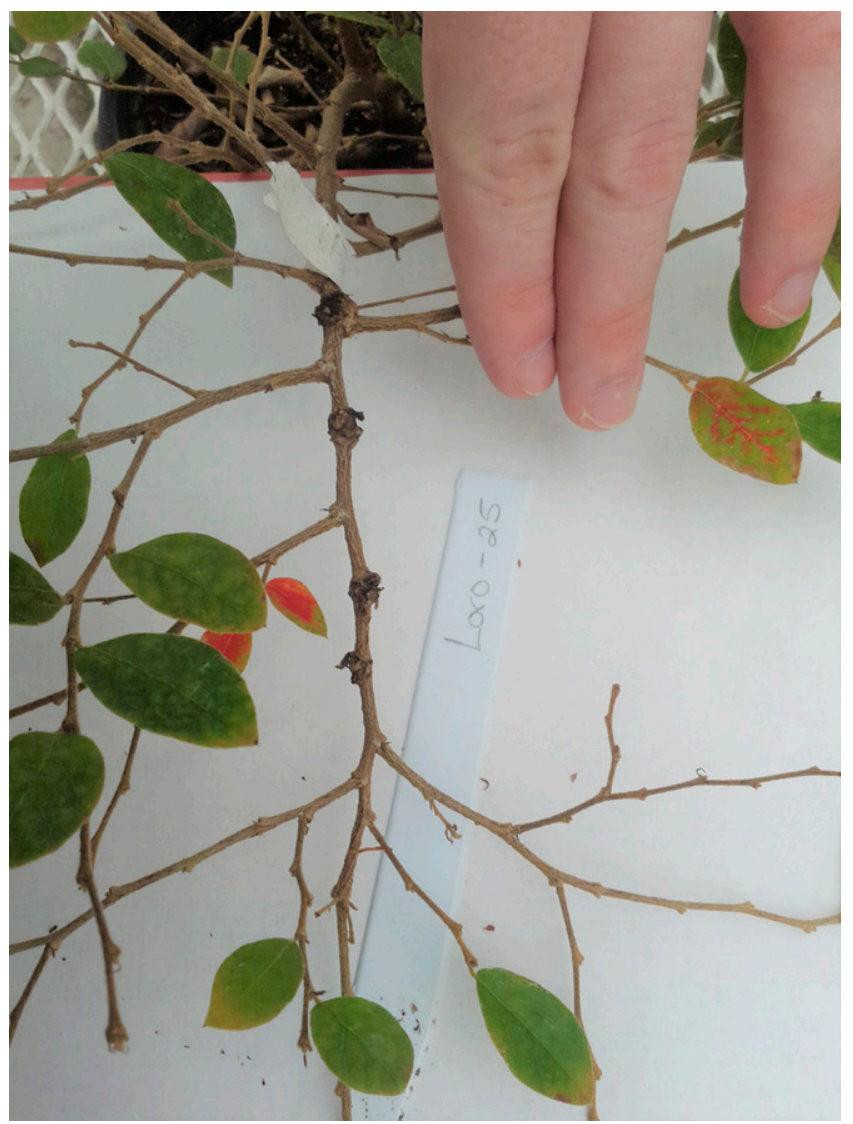

Fig. 5. Injection inoculations of bacterial suspension of the Florida strains resulted in galls, tip dieback, and leaf drop after 4 weeks. sequenced (Sanger sequencing, Eurofins MWG Operon, Huntsville, AL) and aligned with reference sequences obtained from NCBI.

For 16S rRNA, MLSA and iaaL sequences obtained via Sanger sequencing, alignment was done using MUSCLE alignment method (Edgar 2004) with the reference sequence available in the NCBI database. Alignments were trimmed to the same size, and sequences were deposited into the GenBank database (https://www.ncbi.nlm. nih.gov) for the following genes: $i a a, 16 \mathrm{~S}$, gyrB, rpoD, gapl, and gltA (accession nos. KM593940 to KM593995) (Table 2). Maximum likelihood phylogenetic trees with 1,000 bootstrap samples were constructed using MEGA (Tamura et al. 2013) and RAxML (Stamatakis 2014). The general time reversible with unequal rates of nucleotide substitution model was used for the analysis.

\section{Results}

Phenotypic characterization of strains. The galls began as cankers at wounds on loropetalum and were dark with rough edges, progressing laterally until they girdled stems, causing branch dieback. The infection did not progress longitudinally or develop systemically, even when the gall was on the main stem or crown, although girdling of the stem at the crown caused death of the plant. Galls were not observed on roots or leaves. Galls were dry, and bacterial streaming was noted rarely, and only from young galled tissue. Colonies that produced a fluorescent pigment were routinely isolated on King's medium B from loropetalum stem galls. The loropetalum strains produced positive reactions for levan production and elicited HRs in tobacco and tomato. All strains were negative for oxidase, potato rot, and arginine dihydrolase activity. On nutrient agar, all the strains produced cream-colored, smooth, round, slightly undulate colonies.

Using BIOLOG, the strains from loropetalum were identified primarily as pathovars of $P$. syringae and $P$. savastanoi and in some cases other pseudomonads (Table 1). Fatty acid analysis of the Florida strains, the Alabama strains, and the reference strains indicated that the Florida strains were nearly identical ( 2 or less Euclidean distance [E.D.]) to each other and to the Alabama strains 12-254 and 13-474 (Fig. 1), but were less than 6 E.D. from P. syringae strains including $P$. syringae pv. eriobotryae. The loropetalum strains were more distant from $P$. savastanoi oleander strain (LMG 5385) and olive strain (LMG 2209 ${ }^{\mathrm{T}}$ ). However, these strains were different from the oleander, olive, $P$. syringae pv. eriobotryae (LMG $2184^{\mathrm{PT}}$ ), and P. meliae (LMG $2220^{\mathrm{T}}$ ) strains. Fatty acid composition of the strains analyzed in this study were compared and the fatty acid composition of $P$. amygdali from a previously published article by Stead (1992) was used in this study. It is clear that the major fatty acids were present in all strains with similar percentages in all strains with the exception of $P$. amygdali (Table 3 ).

Sequence analyses. Sequence analysis of a 16S rRNA for a subset of loropetalum strains revealed high sequence identity with $P$. syringae pv. eriobotryae, $P$. amygdali, $P$. meliae, and $P$. savastanoi pv. savastanoi. Phylogenetic analysis of $16 \mathrm{~S}$ rRNA sequences of Pseudomonas strains isolated from loropetalum and other fluorescent pseudomonads showed that the representative loropetalum strains were identical to each other and to the pathotype strain of $P$. syringae pv. eriobotryae (Fig. 2). The strains grouped with $P$. syringae pv. eriobotryae and were slightly different from other members of genomospecies 2 identified by Gardan et al. (1999) including $P$. amygdali and $P$. savastanoi pv. savastanoi strains isolated from olive and oleander. Based on the ia gene sequences, the loropetalum strains were identical to each other and were different from the P. syringae pv. eriobotryae LMG $2184^{\mathrm{PT}}$ (Fig. 3).

For MLSA, phylogenetic analysis using concatenated nucleotide sequences of four housekeeping genes ( $g a p 1$, gyrB, gltA, and $r p o D$ ) of representative loropetalum strains and representative strains of all genomospecies resulted in a tree derived from the concatenated housekeeping gene sequences. The loropetalum strains had identical sequences and grouped with genomospecies 2 strains and were very close to $P$. amygdali, $P$. meliae, and $P$. syringae pv. eriobotryae and $P$. syringae pv. myricae (Fig. 4). More distant from the loropetalum strains in genomospecies 2 were strains more closely related to $P$. savastanoi including $P$. savastanoi pv. phaseolicola. 
Pathogenicity tests. In inoculation studies, the loropetalum strains elicited galls as shown in Figures 5 and 6 only in loropetalum (Table 4). No galls were observed on wounds from the water inoculations on any of the hosts tested. Inoculations of all the strains isolated from loropetalum in Table 1 resulted in galls on loropetalum only (Table 4). Inoculations of the following pathotype and type strains listed in Table 1, which included $P$. meliae, $P$. syringae pv. eriobotryae, $P$. savastanoi pv. savastanoi, $P$. tremae, and $P$. amygdali, did not produce galls on loropetalum (Table 4 and Fig. 6). Inoculation of the type or pathotype strains for the almond, oleander, and olive pathogens onto their respective hosts resulted in galls only on their respective hosts (Table 4). Additional strains listed in Table 1, which included $P$. savastanoi pv. glycinea, $P$. savastanoi pv. nerii, P. ficuserectae, $P$. syringae pv. ciccarone,$P$. syringae $\mathrm{pv}$. myricae, $P$. syringae $\mathrm{pv}$. ulmi, $P$. syringae pv. esculi, $P$. syringae $\mathrm{pv}$. hibisci, $P$. syringae $\mathrm{pv}$. tabaci,
$P$. syringae pv. cunninghamiae, and $P$. syringae pv. rhaphiolepidis, were only inoculated on loropetalum and did not produce any visible galls. The strains isolated from loropetalum produced average sized galls of $12 \mathrm{~mm}$ and the bacterial strains were reisolated from galls and verified by rep-PCR. On the other hand, the wounds on stems inoculated with all other strains didn't extend beyond $4 \mathrm{~mm}$. Thus, the only strains causing galls on loropetalum were originally isolated from loropetalum (Fig. 6).

\section{Discussion}

We consistently isolated a fluorescent pseudomonad from galls associated with loropetalum. The bacterium was typical of the $P$. syringae complex (Lelliott et al. 1966) based on LOPAT tests. Fatty acid and Biolog analyses also placed the loropetalum strains in the $P$. syringae complex. Both techniques provided more

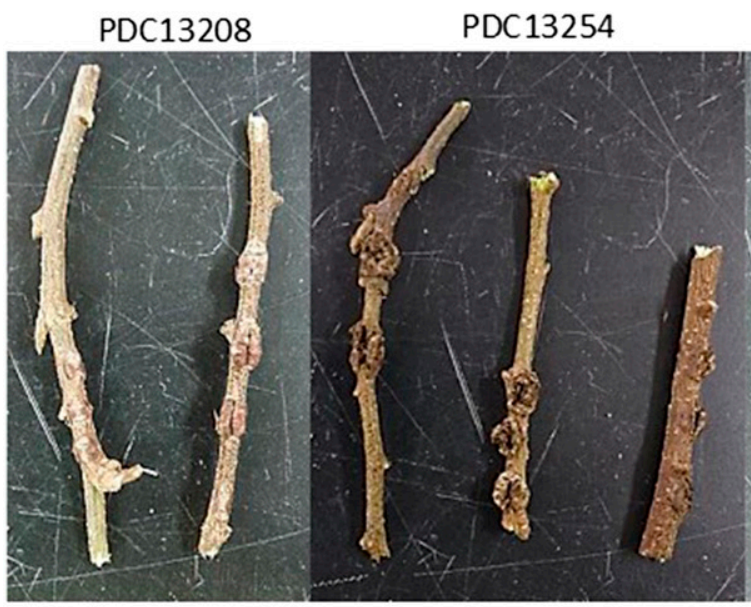

\section{PDC13206}

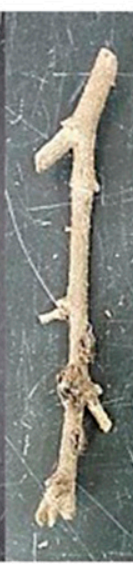

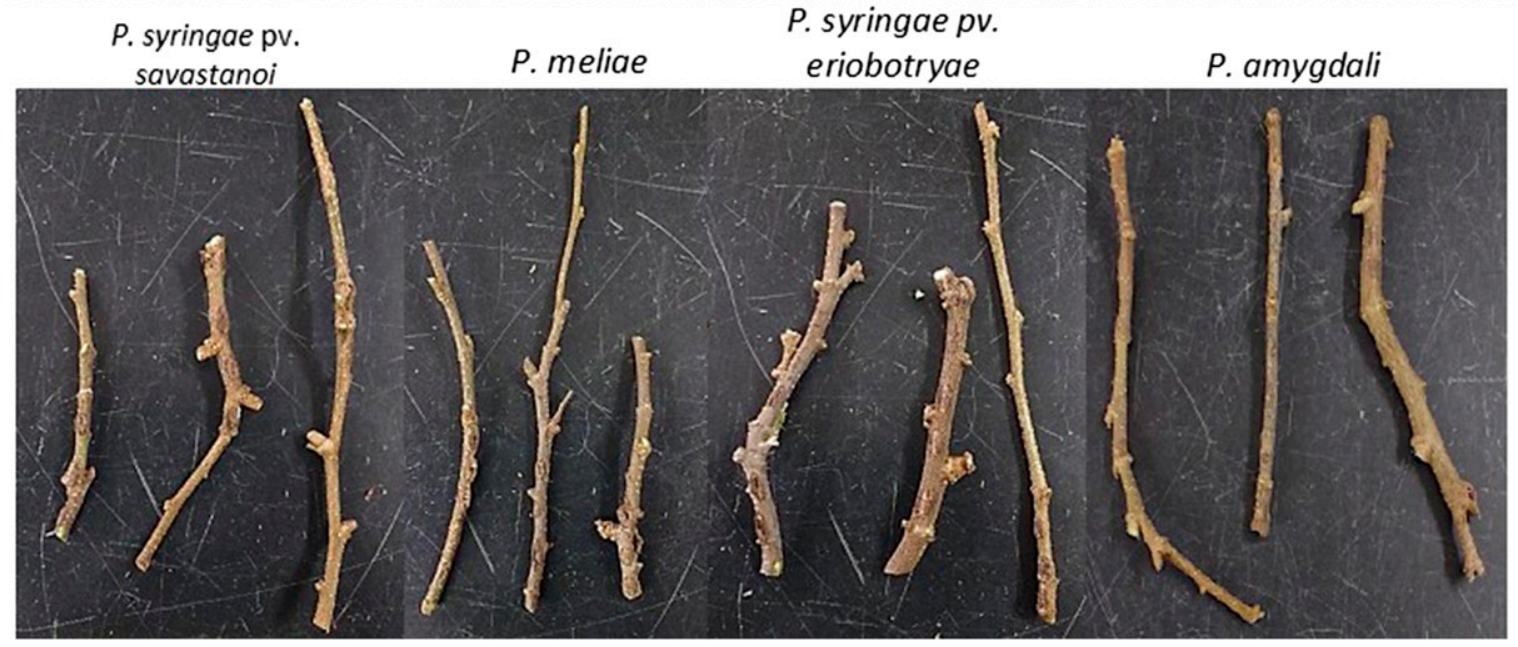

Fig. 6. Pathogenicity on Loropetalum chinense 6 weeks after inoculation. Only the loropetalum strains (upper panels) caused galls on the loropetalum. Pathotype strains of $P$. amygdali did not cause galls on this host (lower panels).

Table 4. Pathogenicity of select strains on almond, loropetalum, mandevilla, oleander, and olive ${ }^{\text {a }}$

\begin{tabular}{|c|c|c|c|c|c|}
\hline Strain & Almond & Loropetalum & Mandevilla & Oleander & Olive \\
\hline PDC12 254A & - & + & - & - & - \\
\hline PDC13-198 to PDC13-228b & - & + & - & - & - \\
\hline PDL384A & - & + & - & - & - \\
\hline LMG 2184 & - & $+/-$ & - & - & - \\
\hline LMG 2209 & - & - & - & - & + \\
\hline LMG 2220 & - & - & - & - & - \\
\hline LMG 5385 & - & - & - & + & - \\
\hline LMG 22121 & - & - & N/A & - & _- \\
\hline LMG 13184 & + & - & N/A & - & _- \\
\hline
\end{tabular}

\footnotetext{
$\mathrm{a}_{+}=$Positive reaction; $-=$no disease reaction; $+/-=$slight reaction; N/A $=$not tested.
}

${ }^{\mathrm{b}}$ Includes the pathotype strain PDC13-208 ${ }^{\mathrm{PT}}$ (DSMZ $105780^{\mathrm{PT}}$ ). 
comprehensive phenotypic information on the strains. Pathogenicity tests confirmed that the loropetalum strains are specific to loropetalum and not pathogenic on almond, mandevilla, oleander, or olive. Furthermore, none of the other gall-producing species or pathovars produced galls on loropetalum although $P$. syringae pv. eriobotryae produced a slight reaction on loropetalum.

Sequence analysis of the 16S rRNA placed the selected strains most closely with $P$. syringae pv. eriobotryae and slightly more distant from the validly published species $P$. savastanoi, $P$. meliae, and the type strain of $P$. amygdali. In MLSA using the four housekeeping genes, the loropetalum strains clustered most closely with $P$. amygdali, were slightly different from $P$. meliae, and more distant from $P$. savastanoi strains. Phylogenetic analysis of the iaa target of the Florida strains, the published Alabama strain (strain PDL384), and the LMG type strains indicated that the loropetalum strains are different from the $P$. savastanoi iaa gene.

Gardan et al. (1999) identified nine genomospecies of which genomospecies 2 consisted of syringae pathovars including lachrymans, eriobotryae, morsprunorum, and nomenspecies $P$. amygdali, $P$. meliae, $P$. savastanoi, and $P$. ficuserectae. Of those, $P$. amygdali is the earliest valid name for genomospecies 2. Given the DNA-DNA hybridization results of Gardan et al. (1999), the species listed above along with those pathovars should be considered synonymous and the correct name for the species should be $P$. amygdali due to priority. Although $P$. amygdali could not be differentiated based on phenotypic tests, it is a validly published name and thus the new pathovar we are describing as well as other organisms in genomospecies 2 should be referred to as $P$. amygdali.

Therefore, based on physiological and biochemical tests including fatty acid analysis, BIOLOG, 16S rRNA sequencing, and more importantly MLSA and pathogenic specificity to infect loropetalum, we have identified a homogeneous group of strains that based on molecular approaches are more closely related to $P$. amygdali than to $P$. savastanoi as previously published (Conner et al. 2013). As such we, propose that this organism be identified as a new pathovar of $P$. amygdali and propose the name $P$. amygdali pv. loropetali $\mathrm{pv}$. nov. and PDC13-208 (change to: DSM 105780) as the pathotype strain. The fatty acid profiles for the loropetalum strains were very distinct from those of $P$ amygdali, as indicated by Stead (1992). However, since none of the strains within $P$. amygdali have the same values as $P$. amygdali in Table 3, the loropetalum values are not out of the range of all $P$. amygdali strains. Stead (1992) noted large quantitative differences in certain fatty acids between $P$. amygdali and $P$. syringae and close relatives. Although qualitatively they contained similar fatty acids with minor exceptions, these differences in composition observed in $P$. amygdali may result from its slow growth (Stead 1992).

Although the loropetalum pathogens were first isolated in diagnostic laboratories, the efforts required to clarify the identity of this pathogen of loropetalum are far beyond the normal range of work involved in a diagnostic laboratory's determination; this work is more along the lines of confirmatory identification. However, it was important to determine that the host range does not include olive since Florida is a state with a growing olive industry. For now, recognizing that $P$. amygdali pv. loropetali pv. nov is not a pathogen of olive nor oleander will help define its management in the ornamental trade in Florida and likely other states. The distinctive host range of the loropetalum strains, supported by the molecular assays, indicates that these strains represent a new pathovar, P. amygdali pv. loropetali $\mathrm{pv}$. nov., which is proposed below.

Description of Pseudomonas amygdali pv. loropetali pv. nov. (loropetali pertaining to Loropetalum, the generic name of the host plant, Loropetalum chinense). Description as for P. amydali. On nutrient agar as well as on KMB, the colonies were color cream, smooth, and round but slightly undulate. Strains fluoresced on KMB. In LOPAT tests, strains were positive for levan production and tobacco hypersensitive reaction, and negative for oxidase arginine dihydrolase activity and ability to rot potato, which identifies the strains as $P$. syringae. MLSA of concatenated genes $g y r \mathrm{~B}, r p o \mathrm{D}$, gapl, and gltA place the strains in genomospecies 2-Group A of
$P$. amygdali. Differentiated from other pathovars by gall formation on loropetalum (L. chinense; natural host) and because it is not pathogenic on almond, mandevilla (Mandevilla sanderi), oleander (Nerium oleander), and olive (Olea europaea). The pathotype strain is designated as PDC13-208 (= DSMZ $105780^{\mathrm{PT}}$ ).

\section{Acknowledgments}

The authors thank E. Dickstein, A. Jeyaprakash, G. Minsavage, A. Ryan, V. Soltanov, A. Vitoreli, and J. Yarbrough for their valuable assistance with the technical aspects of this research, and Professor Nancy de Grummond (Florida State University) for her expert advice on the Latinization of the specific epithet for the organism.

\section{Literature Cited}

Bull, C. T., Clarke, C. R., Cai, R., Vinatzer, B. A., Jardini, T. M., and Koike, S. T. 2011. Multilocus sequence typing of Pseudomonas syringae sensu lato confirms previously described genomospecies and permits rapid identification of $P$. syringae pv. coriandricola and $P$. syringae pv. apii causing bacterial leaf spot on parsley. Phytopathol. 101:847-858.

Bull, C. T., and Koike, S. T. 2015. Practical benefits of knowing the enemy: modern molecular tools for diagnosing the etiology of bacterial diseases and understanding the taxonomy and diversity of plant-pathogenic bacteria. Annu. Rev. Phytopathol. 53:157-180.

Coenye, T., and Vandamme, P. 2003. Intragenomic heterogeneity between multiple 16s ribosomal RNA operans in sequenced bacterial genomes. FEMS Microbiol. Lett. 228:45-49.

Conner, K. O., Olive, J., Zhang, L., Jacobi, J., and Putnam, M. L. 2013. First report of bacterial gall on Loropetalum chinense caused by Pseudomonas savastanoi in the United States. Plant Dis. 97:835.

Edgar, R. C. 2004. MUSCLE: multiple sequence alignment with high accuracy and high throughput. Nucleic Acids Res. 32:1792-1797.

Gardan, L., Bollet, C., Abu Ghorrah, M., Grimont, F., and Grimont, P. A. D. 1992. DNA relatedness among the pathovar strains of Pseudomonas syringae subsp. savastanoi Janse (1982) and the proposal of Pseudomonas savastanoi sp. nov. Int. J. Syst. Bacteriol. 42:606-612.

Gardan, L., Shafik, H., Belouin, S., Broch, R., Grimont, F., and Grimont, P. A. D 1999. DNA relatedness among the pathovars of Pseudomonas syringae and description of Pseudomonas tremae sp. nov. and Pseudomonas cannabina sp. nov. (ex. Sutic and Dowson 1959). Int. J. Syst. Bacteriol. 49:469-478.

Glickmann, E., Gardan, L., Jacquet, S., Hussain, S., Elasri, M., Petit, A., and Dessaux, Y. 1998. Auxin production is a common feature of most pathovars of Pseudomonas syringae. Mol. Plant-Microbe Interact. 11:156-162.

Hwang, M. S. H., Morgan, R. L., Sarkar, S. F., Wang, P. W., and Guttman, D. S. 2005. Phylogenetic characterization of virulence and resistance phenotypes of Pseudomonas syringae. Appl. Environ. Microbiol. 71:5182-5191.

Janda, M. J., and Abbott, S. L. 2007. 16S rRNA gene sequencing for bacterial identification in the diagnostic laboratory: Pluses, perils, and pitfalls. J. Clin. Microbiol. 45:2761-2764.

Lelliott, R. A., Billing, E., and Hayward, A. C. 1966. A determinative scheme for the fluorescent plant pathogenic Pseudomonads. J. Appl. Bacteriol. 29:470-489.

Marchesi, J. R., Sato, T., Weightman, A. J., Martin, T. A., Fry, J. C., Hiom, S. J., and Wade, W. G. 1998. Design and evaluation of useful bacterium-specific PCR primers that amplify genes coding for bacterial 16s rRNA. Appl. Environ. Microbiol. 64:795-799.

Marques, A. S. A., Marchaison, A., Gardan, L., and Samson, R. 2008. BOX-PCR based identification of bacterial species belonging to Pseudomonas syringa- $P$. viridiflava group. Genet. Mol. Biol. 31:106-115.

Parkinson, N., Bryant, R., Bew, J., and Elphinstone, J. 2011. Rapid phylogenetic identification of members of the Pseudomonas syringae species complex using the rpoD locus. Plant Pathol. 60:338-344.

Penyalver, R. G., Garica, A., Ferrer, A., Bertolini, E., and Lopez, M. M. 2000 Detection of Pseudomonas savastanoi pv. savastanoi in olive plants by enrichment and PCR. Appl. Environ. Microbiol. 66:2673-2677.

Ramos, C., Matas, I. M., Bardaji, L., Aragon, I. M., and Murillo, J. 2012. Pseudomonas savastanoi pv. savastanoi: some like it knot. Mol. Plant Pathol. 13:998-1009.

Sarkar, S. F., and Guttman, D. S. 2004. Evolution of the core genome of Pseudomonas syringae, a highly clonal, endemic plant pathogen. Appl. Environ. Microbiol. 70: 1999-2012.

Schaad, N., Jones, J., and Chun, W. (eds.). 2001. Laboratory Guide for Identification of Plant Pathogenic Bacteria (3rd ed.). APS Press, St. Paul, MN.

Stamatakis, A. 2014. RAxML Version 8: A tool for phylogenetic analysis and post-analysis of large phylogenies. Bioinformatics 30:1312-1313.

Stead, D. E. 1992. Grouping of plant-pathogenic and some other Pseudomonas spp. by using cellular fatty acid profiles. Int. J. Syst. Bact. 42:281-295.

Tamura, K., Stecher, G., Peterson, D., Filipski, A., and Kumar, S. 2013. MEGA6: Molecular Evolutionary Genetics Analysis Version 6.0. Mol. Biol. Evol. 30: 2725-2729.

Young, J. M. 2010. Taxonomy of Pseudomonas syringae. J. Plant Pathol. 92: S5-S14. 